\title{
¿Hacia un gobierno transfronterizo? Explorando la institucionalidad para la "integración" colombo-peruana
}

\section{Towards a cross-border government? Exploring institutionality for Colombo-Peruvian "integration"}

Recibido el 21 de octubre de 2015. Aceptado el 18 de agosto de 2016.

*Autor para correspondencia: Jorge Aponte Motta, correo electrónico: apontemotta@gmail.com

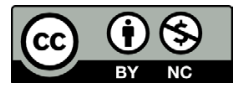

Todos los contenidos de Estudios Fronterizos se publican bajo la licencia Creative Commons Atribución no comercial 2.5 México, y pueden ser usados gratuitamente para fines no comerciales, dando el crédito a los autores y a la revista Estudios Fronterizos.
Adriana Mayela Hurtado Bautista ${ }^{a}$ (http://orcid.org/0000-0003-4392-4767) Jorge Aponte Motta ${ }^{a^{*}}$ (http://orcid.org/0000-0002-4844-2727)

\footnotetext{
a Universidad Nacional Sede Amazonia, Grupo de Estudios Transfronterizos, Bogotá, Colombia, correos electrónicos: amhurtadob@unal.edu.co, apontemotta@gmail.com
}

\section{Resumen}

Este artículo explora la dinámica de la cooperación fronteriza entre Perú y Colombia. Analiza brevemente algunos de los mecanismos de cooperación e indica, a partir de informes técnicos, análisis normativo y organizacional, que estos fracasaron entre otras cosas, por haber articulado fragmentariamente a los entes locales e intermedios de gobierno en la gestión de la cooperación binacional. Dicha dificultad se presenta incluso en las adaptaciones más recientes de los mecanismos de cooperación, lo cual augura un nuevo fracaso en los esfuerzos de ambos países. Por tanto, es necesario ensayar otra forma de ejercicio de la diplomacia en la cual los entes locales e intermedios de gobierno tengan papeles centrales en la cooperación binacional, en aras de enfrentar las problemáticas estructurales e institucionales constantes en el diseño de las políticas, aplicando un enfoque transfronterizo.

Palabras clave: cooperación transfronteriza, cooperación binacional, relaciones internacionales.

\section{Abstract}

This article explores the dynamic of border cooperation between Peru and Colombia. It briefly analyzes some of the cooperation mechanisms and indicates, based on technical reports as well as normative and organizational analysis, that these cooperation mechanisms failed due to, among other reasons, having articulated the local and intermediary government entities in a fragmented manner in managing binational cooperation. This difficulty is even present in the most recent adaptations of the cooperation mechanisms, predicting a new failure in the efforts of both countries. Therefore, it is necessary to attempt a different man-

CÓMO CITAR: Hurtado, A. M. y Aponte, J. (2017). ¿Hacia un gobierno transfronterizo? Explorando la institucionalidad para la "integración" colombo-peruana [Towards a cross-border government? Exploring the institutionality for Colombo-Peruvian "integration"]. Estudios Fronterizos, 18(35), 70-89, doi: 10.21670/ref.2017.35.a04 
ner of exercising diplomacy, in which the local and intermediary government entities play central roles in binational cooperation, in the interest of facing the structural and institutional problems established in the design of these policies, applying a cross-border focus.

Keywords: cross-border cooperation, binational cooperation, international relations.

\section{Introducción ${ }^{1}$}

La frontera colombo-peruana, tiene una larga historia de tensiones y abandono. Su lejanía e incomunicación se suman al recuerdo del conflicto colombo-peruano, la idea de ser retaguardia de la insurgencia colombiana y el narcotráfico. Estos elementos se adicionan a la precaria atención de las necesidades sociales de sus habitantes, mayoritariamente indígenas. Lo anterior parece contrastar con los intensos esfuerzos de ambos Estados por desarrollar: $a$ ) políticas de frontera que pretenden ser cada vez más incluyentes, $b$ ) el fortalecimiento de las relaciones bilaterales y $c$ ) conseguir la proliferación de acuerdos multilaterales de cooperación en los que participan los dos países.

A pesar de todos estos desarrollos, los diseños de políticas públicas y las redes institucionales para su implementación, no hay correspondencia con lo que sucede en la frontera. Parece haber cierta descoordinación entre la evolución legislativa, de documentos que ofrecen lineamientos de políticas y su efectiva implementación local. Lo anterior sugiere cierta inercia en las políticas de frontera y cooperación colomboperuanas. Como en el mito de Sísifo, la región fronteriza colombo-peruana carga la piedra de una organización administrativa que no responde a las demandas de gobierno. Sísifo se esfuerza enteramente por subir la cuesta de la montaña, con el sino trágico de arrancar en el mismo punto cada día. El eterno retorno de lo inútil de una cooperación que no sale del papel y de una institucionalidad que no da cabida a lo local es la tragedia de la cooperación fronteriza colombo-peruana².

En este artículo analizamos brevemente algunos mecanismos de cooperación existentes entre Colombia y Perú, resaltando que varias de las dificultades de estos mecanismos para cumplir sus objetivos de fomentar la cooperación y el desarrollo, se relacionan con el diseño institucional nacionalizado que bloquea la real cooperación transfronteriza. En este sentido, sugerimos que es pertinente adelantar estrategias organizativas tendientes a una efectiva cooperación a través de la frontera, o si se quiere, un gobierno transfronterizo que supere las dificultades que impone la comprensión nacionalizada de las relaciones internacionales. Apostamos por tanto, por la consolidación de un nuevo tipo de diplomacia que parta desde lo local para fortalecer las redes de cooperación transfronterizas.

En esta línea, sin entrar en discusión sobre los procesos de integración y cooperación en Europa, Norteamérica o Sur América, ${ }^{3}$ planteamos la discusión desde la particularidad

\footnotetext{
${ }^{1}$ Este artículo es un resultado preliminar del Proyecto de Investigación adelantado por el Grupo de Estudios Transfronterizos-GET de la Universidad Nacional de Colombia Sede Amazonia, denominado: "Ciudades y poblaciones fronterizas: Una propuesta hacia la armonización de las políticas públicas en la frontera internacional del Departamento del Amazonas con Brasil y Perú", financiado por el Departamento de Amazonas y Colciencias con apoyo de recursos del Banco Interamericano de Desarrollo. Las opiniones son responsabilidad de los autores.

${ }^{2}$ La inspiración para realizar la analogía al mito de Sísifo, la debemos al trabajo de Darío Restrepo (2001).

${ }^{3}$ Sugerimos como elementos para el debate que no constituyen un estado del arte, las siguientes lecturas: para la zona de frontera México-Estados Unidos lecturas de Peña (2004) y García (2015); para la Unión
} 
de las organizaciones políticas e institucionales colombianas y peruanas, acerca de la necesidad de adaptaciones organizacionales e institucionales de carácter transfronterizo para gobernar la frontera, saliendo de las lógicas administrativas y organizativas de los Estados nacionales desde donde tradicionalmente se ha entendido el gobierno y la administración fronteriza.

Así, acudimos a la propuesta de construir gobernanzas transfronterizas, lo cual implica el desarrollo de arreglos político-administrativos y de gobierno que integren la dimensión fronteriza en su configuración (Sausi y Oddone, 2012, p. 241), ${ }^{4}$ así como desarrollar esquemas de diplomacia local o paradiplomacia (Oddone y Rodríguez, 2015), que trasciendan los marcos más clásicos de desarrollo de las relaciones internacionales dentro de los cuales muchas veces se han comprendido las fronteras, borrando en este camino su particularidad local, para comprender su administración desde los niveles locales de gobierno.

\section{Acuerdos binacionales: Cooperación en el papel, prácticas descoordinadas}

Colombia y Perú hacen parte de mecanismos multilaterales y han firmado numerosos acuerdos bilaterales tendientes a ampliar la cooperación en un camino hacia la integración regional, meta que es incluida en la constitución de ambos países (Constitución Política de Colombia de 1991, preámbulo, arts. 9 y 227; Landa y Velazco, 2007). Pese a lo anterior, las dificultades y asimetrías en la implementación local de los acuerdos interestatales, demuestran una escasa articulación local transfronteriza, haciendo que las problemáticas persistan sin una solución real.

Es importante resaltar el intenso desarrollo que en años recientes ha tenido la relación bilateral en el marco de la Comunidad Andina de Naciones (CAN), a través de la adopción de la Política Comunitaria para la Integración y el Desarrollo Fronterizo, acogida formalmente por medio de las decisiones 459, 501 y 502 de la can. De esto se desprende también la creación de la Zona de Integración Fronteriza Colombo-Peruana (ZIF-CP) a partir de 2002, la cual se ve fortalecida por la inclusión en la legislación de ambos países de figuras de ordenación que involucran formas de integración territorial supranacional. Sin embargo, el desarrollo de tal zIF, se enmarca en los criterios establecidos en las relaciones bilaterales. Así, Perú además de la zif con Colombia, se encuentra adelantando de manera independiente una zIF con Brasil (Acuerdo Marco entre la República del Perú y República Federativa del Brasil para el establecimiento de la "Zona de Integración Fronteriza Perú-Brasil”, 2009) mientras Colombia, adelanta otra con dicho país, ${ }^{5}$ lo cual refleja el amarre bilateral del desarrollo de los mecanismos de integración y afecta la institucionalización de mecanismos trinacionales. ${ }^{6}$

Europea Oliveras, Durà y Perkmann, (2010), Coletti (2010) y Anderson, O'Dowd y Wilson (2003). Finalmente para las experiencias latinoamericanas, en concreto en el Cono Sur: Ferrero (2006), Moreno (2012) y Sausi y Oddone (2010).

${ }^{4}$ Aunque es importante indicar que hay que mirar con más precaución el efecto de las propuestas de integración física, actualmente en intenso desarrollo en Sur América, así como de la llamada "nueva geografía económica" en las cuales los citados autores depositan grandes esperanzas.

${ }^{5}$ La vecindad colombo-brasileña se constituyó en 1993. Sus últimas acciones han estado relacionadas con el establecimiento de un régimen especial, promulgado en la Ley 1463 del 29 de junio de 2011 (Ley 1463 de 2011) y reglamentado mediante el Decreto 155 de 2014, que establece una Zona de Régimen Especial Fronterizo, sobre la cual aún hay gran expectativa de cómo llegue a desarrollarse (Decreto 155 de 2014).

${ }^{6}$ Esta es una de las conclusiones que pronto serán publicadas del proyecto que sustentó este artículo. Dicho documento recomienda fortalecer mecanismos de cooperación trinacional para romper el bilateralismo que bloquea los acuerdos locales (Aponte, Victorino y Zárate, en prensa). 
Por otra parte, pese al reciente acuerdo de los ministros de relaciones exteriores de Colombia y Perú que esboza la posible estructura funcional para la implementación del tratado en una comisión binacional cuya cabeza y cuerpo se mueve a través de los ministerios de relaciones exteriores, con cierto nivel de desconcentración en la parte peruana, y una presencia nominal de los niveles intermedios de gobierno, (Acuerdo para la implementación del Plan de Desarrollo de la Zona de Integración Fronteriza entre la República de Colombia y la República de Perú, 2014) aún no es clara la operatividad funcional y presupuestal para adelantar dicha ZIF, lo cual podría conducir a adelantar esfuerzos nacionales descoordinados hacia el área de atención fronteriza sin realmente poder articularse y generar estrategias de cooperación e integración efectivas. Dicho camino parece ser el que siguieron varios de los mecanismos de cooperación entre ambos países; por mencionar dos de ellos: el Convenio de Cooperación Aduanera entre la República de Colombia y la República Peruana (CCA) de 1938 y el Plan Colombo Peruano para el desarrollo integral de la cuenca del río Putumayo (РPCP) de 1998.

El primero surgió en el marco del Protocolo de Amistad y Cooperación firmado en Río de Janeiro en 1934 (1934, artículo 4 y artículo 14 del acta adicional), que dio fin al conflicto entre ambos países entre 1932 y 1933. El cCA de 1938, estableció un listado de productos que debían gozar de un arancel común en el área de dicho convenio. Este fue ajustado a las nuevas condiciones del Pacto Andino con el Protocolo Modificatorio del cCA de 1938 (1981) el cual contempló, en su artículo Ix, un Grupo Mixto de Estudio del mecanismo, lo que se tradujo en el marco de la iv Reunión de la Comisión Mixta Colombo-Peruana de Cooperación Amazónica efectuada en 1990, en la creación de una Comisión Bilateral Administradora del CCA; en la cual sendas comisiones administradoras nacionales evaluarían el desarrollo e implementación del acuerdo aduanero.

La Comisión Administradora Nacional Colombiana fue creada en 1992 (Decreto 0211, 1992), pero su labor de revisión ha sido escasa, así como su continuidad en el marco de las relaciones bilaterales. Tanto es así que dicha Comisión Bilateral no fue incluida entre las instancias de coordinación contempladas en el Plan de la Zona de Integración Fronteriza Perú-Colombia, pese a que dicho plan considera importantes reformas al Convenio; esto indica que la institucionalización de la Comisión del Convenio Aduanero se quedó en los propósitos iniciales (Proyecto Especial para el Desarrollo de la Cuenca del Río Putumayo [Pedicp], 2013).

Sin embargo, es importante resaltar que en dicha comisión, aparte de los ministerios de comercio y relaciones exteriores, tuvieron asiento los gobernadores de Amazonas y Putumayo, así como las cámaras de comercio de ambos departamentos, lo cual permite entrever cierta participación local en el mecanismo (Decreto 0211, 1992).

A pesar de lo anterior, es una crítica constante de los comerciantes en la región, la estrechez del acuerdo aduanero y su inadaptación a las condiciones actuales del comercio que lo hacen poco operativo y motivan una posible transformación del mecanismo. ${ }^{7}$

Por su parte, el PPCP surgió en el marco del Tratado de Cooperación Amazónica Colombo Peruano de 1979. Para el año de 1988, ambos países suscribieron una declaración conjunta en donde acordaron un Plan de Acción Bilateral para el desarrollo de la región amazónica que comparten (Plan Colombo-Peruano para el Desarrollo Integral de la Cuenca del Río Putumayo-Diagnóstico Regional Integrado, 1993).

\footnotetext{
${ }^{7}$ Tal como fue comentado en el desarrollo de los talleres realizados en las localidades pares fronterizas de Leticia-Tabatinga, Puerto Nariño-Caballo Cocha, Tarapacá-Ipiranga, Pedrera-Vila Bittencourt, Puerto Leguízamo-Soplin Vargas, El Estrecho durante el segundo semestre de 2013 en el marco del proyecto que amparó este trabajo.
} 
Sus objetivos se dirigieron a la atención de las poblaciones aisladas asentadas en la cuenca del río Putumayo, que presentaban debilidad institucional, carencia de intervención estatal y bajo desarrollo económico y social. En este sentido, se plantearon como objetivos: a) promover el desarrollo sostenido de la zona, $b$ ) mejorar el nivel de vida de la población, $c$ ) lograr integrar la zona con el territorio de ambos países, para lo cual era necesario construir la infraestructura de vías de comunicación y medios de transporte. Además, como la mayor parte de la población asentada en la cuenca del río Putumayo pertenece a comunidades indígenas, se debían priorizar las acciones dirigidas a ellos, promoviendo la protección de derechos y asegurando su desarrollo integral social y cultural (Plan Colombo-Peruano para el Desarrollo Integral de la Cuenca del Río Putumayo-Diagnóstico Regional Integrado, 1993).

En Colombia comprendía los departamentos de Amazonas y Putumayo teniendo como puntos limitantes desde Leticia hasta el río Caquetá. Para Perú, abarcaba la zona norte del departamento de Loreto, alcanzando algunas partes de las provincias de Maynas y Ramón Castilla, además de poblaciones asentadas a orillas de los ríos Putumayo, Napo, Amazonas y Yavarí (Figura 1).

Figura 1: Área del Plan Colombo-Peruano para el Desarrollo Integral de la Cuenca del Río Putumayo y el Tratado de Cooperación Amazónica

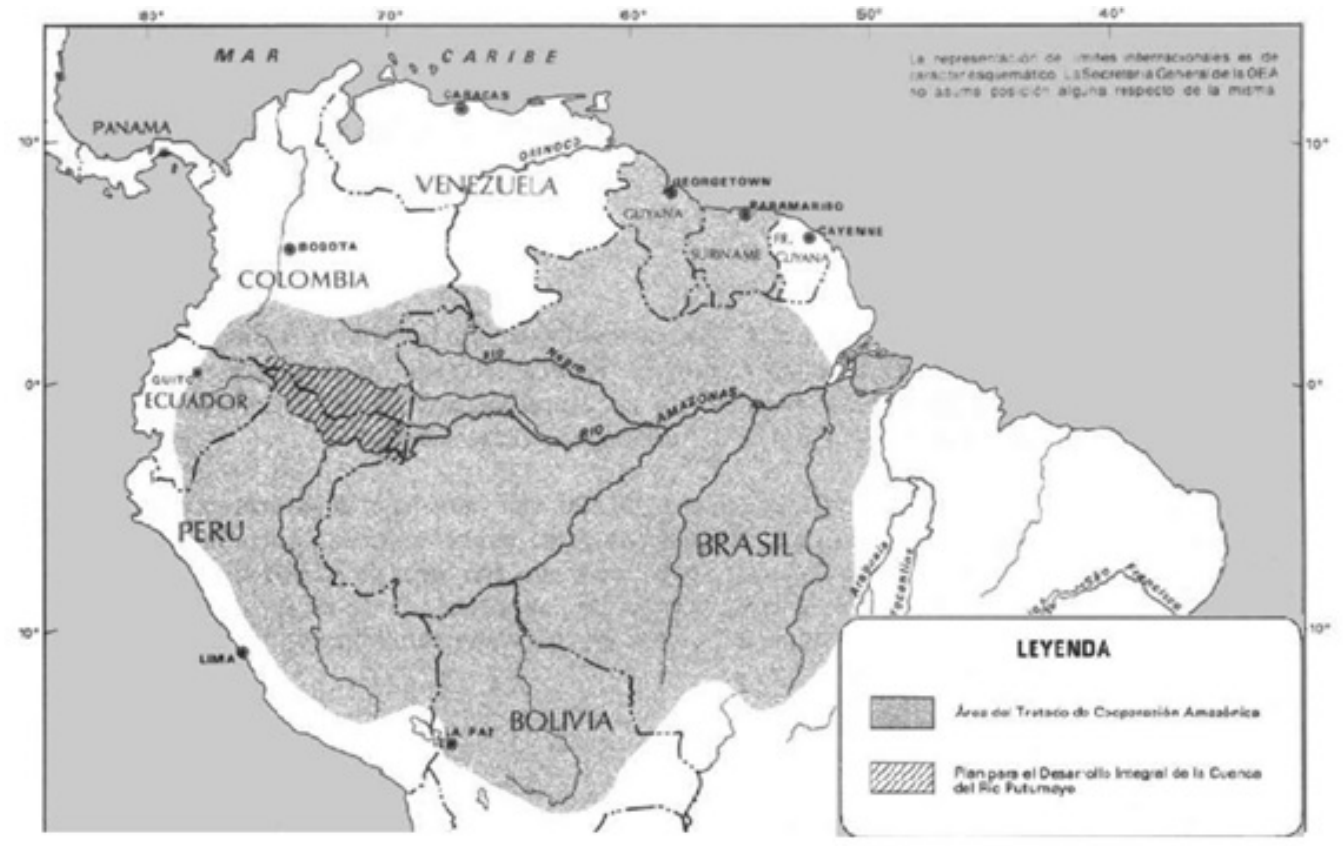

Fuente: Plan Colombo-Peruano para el Desarrollo Integral de la Cuenca del Río Putumayo (PPCP)Diagnóstico Regional Integrado (1993, pp. 73 y 74).

En términos de estructura operativa y de coordinación, el PPCP contempló una comisión ejecutiva articulada a la Comisión Mixta de Cooperación Amazónica dirigida por las cancillerías, un comité técnico binacional de administración y dirección del plan y sendas unidades o coordinaciones técnicas. Para Colombia, inicialmente fue designado el Instituto de Hidrología, Meteorología y Adecuación de Tierras (Himat) y posteriormente (tras su desaparición), el Instituto Amazónico de Investigaciones Científicas (Sinchi) y para Perú inicialmente, el Gobierno Regional de Loreto, siendo reemplazado por el 
Pedicp, inicialmente al interior del Instituto Nacional de Desarrollo (Inade) y tras su desaparición del Ministerio de Agricultura (Informe Internacional de la Auditoría en Cooperación. Plan Colombo Peruano para el Desarrollo Integral de la Cuenca del Río Putumayo, 2008; Plan Colombo-Peruano para el Desarrollo Integral de la Cuenca del Río Putumayo-Diagnóstico Regional Integrado, 1993; Plan Colombo-Peruano para el Desarrollo Integral de la Cuenca del Río Putumayo (PPCP), 1998).

Es importante hacer notar que pese a que la enunciación del área de incidencia del PPCP toma como base a unidades de organización político-administrativas, la participación de estas como órganos de gobierno dentro del plan, es muy escasa. Se indica la participación del Gobierno Regional de Loreto, pero no se llegaron a precisar sus funciones, siendo incluso suplantada por una entidad del orden nacional, el Pedicp. La escasa participación de los entes político-administrativos locales, así como la vinculación pobre de los habitantes de la región, son algunos de los elementos que restringieron el funcionamiento del citado plan, junto a una amplia gama de restricciones presupuestales, administrativas y de auditoría que fueron indicadas por las Entidades de Control Fiscal de Colombia y Perú en un informe conjunto realizado en 2008 (Informe Internacional de la Auditoría en Cooperación. Plan Colombo Peruano para el Desarrollo Integral de la Cuenca del Río Putumayo, 2008).

Entre los elementos más interesantes analizados en el citado informe, resalta la escasa coordinación binacional, la poca itinerancia de las comisiones de alto nivel y el escaso impacto de sus acuerdos, ya que no se tradujeron en acciones efectivas desarrolladas por las comisiones técnicas. Por otra parte, indicó que ambas comisiones tuvieron problemas de adecuación entre sus acciones, enfoques misionales y los objetivos del PPCP, lo cual hizo que los avances en más de 20 años de implementación del plan solo resaltaran en dos proyectos de 11 contemplados y lo más grave, que las condiciones de aislamiento, baja articulación económica, condiciones precarias de la calidad de vida y deterioro medioambiental no lograron superarse (Informe Internacional de la Auditoría en Cooperación. Plan Colombo Peruano para el Desarrollo Integral de la Cuenca del Río Putumayo, 2008, p. 62).

Pese a lo anterior, no se puede subvalorar el trabajo adelantado por ambas instituciones, cada una en su campo. Los desarrollos en infraestructuras y programas de producción adelantados por el Pedicp constituyen avances importantes y los trabajos de diagnóstico e investigación dirigidos desde el Sinchi, son la base para buena parte de las investigaciones que se adelantan regionalmente; sin embargo, los problemas de coordinación, el enfoque nacional de su acción en el marco de un plan diseñado binacionalmente y la escasa participación local, cuestionan dichos logros.

\section{¿Se repiten los errores del Plan Colombo Peruano para el Desarrollo Integral de la Cuenca del Río Putumayo? Articulando por arriba sin participación de los de abajo}

Las experiencias anteriores obligan a pensar que es primordial comprender de forma binacional las posibilidades reales de coordinación que podrían tener no solo estas instituciones de carácter nacional en el marco de la legislación y las políticas de frontera de cada uno de los países, sino también las responsabilidades y mecanismos de articulación políticos y administrativos a escalas locales para fortalecer las estructuras de gestión y administración de la planificación transfronteriza. 
En ese camino, se vislumbra como instancia fundamental de articulación la Comisión de Vecindad Colombo Peruana y en profunda vinculación con esta, las posibles estructuras organizacionales que puedan constituirse con la ZIF, sin embargo estas últimas no se concretan pese a existir una Comisión de Transición del PPCP hacia la ZIF instaurada desde 2009.

La Comisión de Vecindad Colombo Peruana es un mecanismo bilateral constituido en 1994 para dar seguimiento permanente, realizar análisis, proyección de agendas y compromisos, así como establecer la necesidad de adelantar acuerdos bilaterales para facilitar los procedimientos previstos, unificar acciones o formalizar propuestas y actividades en el marco de la relación bilateral. La comisión estaba integrada inicialmente por representantes de ambos gobiernos y por los principales gremios empresariales de ambos países, los cuales, realizarían reuniones periódicas (Ministerio de Relaciones Exteriores, 1994, p. 1).

Dicho instrumento evolucionó en el 2003 a través del mecanismo de canje de notas diplomáticas entre ambos gobiernos, asignándole a esta Comisión, presidida por los cancilleres, el rol de ser la máxima instancia político-diplomática entre ambos países, la cual debería orientar, coordinar, articular y supervisar los mecanismos, programas, proyectos y actividades binacionales que se dieran en los temas específicos de cooperación, desarrollo e integración bilateral, siendo el ámbito fronterizo uno de los escenarios en los cuales las actividades de la Comisión debería incidir de forma importante. Dicha comisión tendría reuniones anuales de forma alternada en ambos países (Canje de notas que constituye un acuerdo sobre la redefinición del rol y estructura de la Comisión de Vecindad e Integración Colombo Peruana al fin de dotarla de mayor operatividad y agilidad en su funcionamiento entre la república de Colombia y Perú, 2003).

Estaría integrada por dos comisiones nacionales, cada una de estas tendría un secretario ejecutivo, comisionados (altos representantes del sector público y privado de cada país) y coordinadores de cada uno de los Comités Técnicos Binacionales (ств), los cuales se encargarían de orientar y revisar el cumplimiento de las decisiones adoptadas al interior de estos. A su vez, serían coordinados por altos funcionarios de las áreas competentes de las cancillerías, con la participación de funcionarios de los sectores nacionales correspondientes y representantes de las zonas de frontera. Los ств mantendrían reuniones de forma periódica (y serían: Desarrollo e Integración Fronteriza, Comercio, Turismo e Inversiones y Cooperación) (Canje de notas que constituye un acuerdo sobre la redefinición del rol y estructura de la Comisión de Vecindad e Integración Colombo Peruana al fin de dotarla de mayor operatividad y agilidad en su funcionamiento entre la república de Colombia y Perú, 2003).

Es interesante señalar que en la práctica estas comisiones de vecindad, a partir del año 2003 reinsertaron la importancia de las autoridades y actores locales (por lo menos la más representativa que es la agremiación de comerciantes) a la hora de conformar la delegación colombiana de la Comisión de Vecindad. Sin embargo, el peso en dicha subcomisión de agentes comerciales nacionales denota que este foro estuvo dirigido más a favorecer a los grandes grupos económicos y no a los habitantes fronterizos. Esto se demuestra al hacer una rápida revisión de los miembros de la citada subcomisión en diferentes momentos desde su rediseño en 2003, cuando representantes de grandes empresas hicieron parte de ella y las adiciones realizadas en 2007, cuando los nuevos miembros no fueron designados en relación con su carácter de representantes gremiales o institucionales, sino a título personal.

Dicha composición se vio cuestionada por el número y vinculación organizacional de los participantes en la x Comisión de Vecindad realizada en 2011, en la cual los 
representantes de los sectores privados brillaron por su ausencia, viéndose un amplio número de representantes de diferentes instituciones ${ }^{8}$ del nivel nacional principalmente, del nivel intermedio, representado por el gobernador de Amazonas y representantes de la sociedad civil local, incluyendo de los cabildos indígenas (ver Tabla 1) (Acta Final. x Reunión de la Comisión de Vecindad e Integración Colombo-Peruana, 2011).

Tabla 1: Miembros de la Subcomisión Colombiana de la Comisión de Vecindad e Integración Colombo-Peruana

\begin{tabular}{|c|c|c|}
\hline Decreto 2791 de 2003 & Sector privado & Sector público \\
\hline & $\begin{array}{l}\text { Javier Gutiérrez } \\
\text { Interconexión Eléctrica S. A. }\end{array}$ & Gobernador Departamento de Amazonas \\
\hline & $\begin{array}{l}\text { Hernando Franco } \\
\text { Industrias Manuelita }\end{array}$ & Gobernador Departamento de Putumayo \\
\hline & $\begin{array}{l}\text { Javier Hoyos } \\
\text { Industrias Bavaria }\end{array}$ & \\
\hline & $\begin{array}{l}\text { Alex Vela } \\
\text { Carvajal y Cía. } \\
\end{array}$ & \\
\hline & $\begin{array}{l}\text { Secretario Cámara de Comer- } \\
\text { cio del Amazonas }\end{array}$ & \\
\hline \multirow[t]{9}{*}{ Decreto 3157 de 2007} & Sector privado & Sector público \\
\hline & Luis Carlos Villegas & \\
\hline & Evardo Murillo & \\
\hline & Lucía González & \\
\hline & Germán Grajales & \\
\hline & Fernando Brito & \\
\hline & Silvia Helena Ramírez & \\
\hline & Juan Camilo Jaramillo & \\
\hline & Mónica Lanzetta & \\
\hline \multirow[t]{11}{*}{ x Reunión CVI Col-Pe 2011} & Sector privado & Sector público \\
\hline & & $\begin{array}{l}\text { Francisco Coy } \\
\text { Viceministra de Relaciones Exteriores }\end{array}$ \\
\hline & & $\begin{array}{l}\text { Margarita Tejada } \\
\text { Director Soberanía Territorial y Desarrollo } \\
\text { Fronterizo-Minrelex } \\
\end{array}$ \\
\hline & & $\begin{array}{l}\text { Carlos Arturo Morales } \\
\text { Dirección Soberanía Territorial y Desarrollo } \\
\text { Fronterizo-Minrelex }\end{array}$ \\
\hline & & $\begin{array}{l}\text { Bibiana Rodríguez } \\
\text { Director de América-Minrelex } \\
\end{array}$ \\
\hline & & $\begin{array}{l}\text { Pilar Cruz } \\
\text { Dirección América- Minrelex }\end{array}$ \\
\hline & & $\begin{array}{l}\text { Diana Carvajal } \\
\text { Dirección América-Minrelex }\end{array}$ \\
\hline & & $\begin{array}{l}\text { Luis Armando Soto } \\
\text { Cooperación suR-SUR-Minrelex }\end{array}$ \\
\hline & & $\begin{array}{l}\text { Carlos Alfredo Carretero } \\
\text { Director Asuntos Culturales-Minrelex }\end{array}$ \\
\hline & & $\begin{array}{l}\text { María Andrea Albán } \\
\text { Coordinador de Integración y Desarrollo } \\
\text { Fronterizo }\end{array}$ \\
\hline & & $\begin{array}{l}\text { Carlos Robles } \\
\text { Directora Cooperación Internacional }\end{array}$ \\
\hline
\end{tabular}

\footnotetext{
${ }^{8}$ En la Comisión hubo un total de 47 representantes de la delegación colombiana frente a ocho representan-
} tes de la delegación peruana, lo cual confirma una asimetría importante entre las delegaciones. 


\begin{tabular}{|c|c|c|}
\hline & & $\begin{array}{l}\text { Sandra Muñoz } \\
\text { Director Pesca y Acuicultura-Minagricultura }\end{array}$ \\
\hline & & $\begin{array}{l}\text { Carolina Camacho } \\
\text { Minagricultura }\end{array}$ \\
\hline & & $\begin{array}{l}\text { Gloria Gaviria } \\
\text { Minagricultura }\end{array}$ \\
\hline & & $\begin{array}{l}\text { Mariela Ortega } \\
\text { Cooperación Internacional-Minprotección }\end{array}$ \\
\hline & & $\begin{array}{l}\text { Carolina Bonilla } \\
\text { Minprotección }\end{array}$ \\
\hline & & $\begin{array}{l}\text { Lina Saavedra } \\
\text { Mincomercio }\end{array}$ \\
\hline & & $\begin{array}{l}\text { Marlene Espitia } \\
\text { Mincomercio }\end{array}$ \\
\hline & & $\begin{array}{l}\text { Mauricio Molano } \\
\text { (Acción Social) }\end{array}$ \\
\hline & & $\begin{array}{l}\text { Luis Armando Arias } \\
\text { Minambiente }\end{array}$ \\
\hline & & $\begin{array}{l}\text { Gloria Contreras } \\
\text { Asuntos Internacionales, Instituto } \\
\text { Colombiano Agropecuario (ICA) }\end{array}$ \\
\hline & & $\begin{array}{l}\text { Argelino Ramírez } \\
\text { Director-ICA }\end{array}$ \\
\hline & & $\begin{array}{l}\text { Fabián Romero } \\
\text { Instituto Colombiano de Desarrollo } \\
\text { Rural-INCODER }\end{array}$ \\
\hline & & $\begin{array}{l}\text { Inés Carlota Carriazo } \\
\text { Instituto Nacional de Salud }\end{array}$ \\
\hline & & $\begin{array}{l}\text { Fernando Bedoya } \\
\text { Asuntos Internacionales, Servicio } \\
\text { Nacional de Aprendizaje (SENA) } \\
\end{array}$ \\
\hline & & $\begin{array}{l}\text { Azur Grisales } \\
\text { SENA }\end{array}$ \\
\hline & & $\begin{array}{l}\text { Juan Zamora } \\
\text { Armada Nacional }\end{array}$ \\
\hline & & $\begin{array}{l}\text { Carlos Ortiz } \\
\text { Armada Nacional }\end{array}$ \\
\hline & & $\begin{array}{l}\text { Cristian Jiménez } \\
\text { Fuerzas Militares de Colombia (FMM) }\end{array}$ \\
\hline & & $\begin{array}{l}\text { Mónica Saavedra } \\
\text { Fuerza Naval del Sur }\end{array}$ \\
\hline & & $\begin{array}{l}\text { Laura Ardila } \\
\text { Ejército Nacional }\end{array}$ \\
\hline & & $\begin{array}{l}\text { Esther Arias } \\
\text { Ejército Nacional }\end{array}$ \\
\hline & & $\begin{array}{l}\text { Juliana Mantilla } \\
\text { Asociación de Productores-Exportado- } \\
\text { res de Frutas y Hortalizas de la Región } \\
\text { de Murcia (Proexport) }\end{array}$ \\
\hline & & $\begin{array}{l}\text { Luz Helena Abello } \\
\text { Instituto de Hidrología, Meteorología } \\
\text { y Estudios Ambientales de Colombia } \\
\text { (IDEAM) }\end{array}$ \\
\hline & & $\begin{array}{l}\text { Paula Rodríguez } \\
\text { CAF-Banco de Desarrollo de América } \\
\text { Latina/Pedicp }\end{array}$ \\
\hline & & $\begin{array}{l}\text { Luisa Fernanda Marín } \\
\text { Ministerio del Interior }\end{array}$ \\
\hline
\end{tabular}




\begin{tabular}{|c|c|c|}
\hline & & $\begin{array}{l}\text { Belén Ojeda } \\
\text { Ministerio del Interior }\end{array}$ \\
\hline & & $\begin{array}{l}\text { Claudia Núñez } \\
\text { Min ambiente }\end{array}$ \\
\hline & & $\begin{array}{l}\text { Olga Lucía Toro } \\
\text { Parques Nacionales }\end{array}$ \\
\hline & & $\begin{array}{l}\text { Edgar Rodríguez } \\
\text { Parques Nacionales }\end{array}$ \\
\hline & & $\begin{array}{l}\text { Luz Marina Mantilla } \\
\text { Sinchi }\end{array}$ \\
\hline & & $\begin{array}{l}\text { Belinda Cucicu Sánchez } \\
\text { Corporación para el Desarrollo } \\
\text { Sostenible del Sur de la } \\
\text { Amazonia-Corpoamazonia }\end{array}$ \\
\hline & & $\begin{array}{l}\text { Olbar Andrade } \\
\text { Gobernador del Amazonas }\end{array}$ \\
\hline & & $\begin{array}{l}\text { Bernardita Arremuy } \\
\text { Asuntos Indígenas-Gobernación } \\
\text { Amazonas }\end{array}$ \\
\hline & & $\begin{array}{l}\text { Claudino Pérez } \\
\text { Delegada Cabildo Indígena Amazonas }\end{array}$ \\
\hline & & $\begin{array}{l}\text { Ando Alipio Cortez } \\
\text { Delegado Cabildo Indígena El Encanto }\end{array}$ \\
\hline & & $\begin{array}{l}\text { Héctor Noriega } \\
\text { Presidente Cámara de Comercio } \\
\text { Amazonas }\end{array}$ \\
\hline
\end{tabular}

Fuente: Elaboración propia a partir de Decreto 2791 de 2003, Decreto 3157 de 2007 y Acta final. x Reunión de la Comisión de Vecindad e Integración Colombo-Peruana, 2011.

Tal variación en la composición de estos foros, podría evidenciar cambios en las agendas o restricciones internas de los mismos para definir la agenda binacional y el desarrollo de la política pública fronteriza, lo cual motivaría el distanciamiento de algunos actores.

Por otra parte, los acuerdos alcanzados en el marco de las comisiones de vecindad, parecen dar continuidad a los programas diseñados en el marco del PPCP y avalan las sugerencias de la Comisión Mixta de Cooperación Amazónica. Particularmente los temas relacionados con aprovechamiento y ordenación de recursos forestales y piscícolas, así como temas de conservación, tuvieron especial visibilidad en la última reunión de la Comisión de Vecindad adelantada en 2011, mientras temas relacionados con el fortalecimiento institucional local, se vieron relegados a un segundo lugar (Acta Final. $\mathrm{x}$ Reunión de la Comisión de Vecindad e Integración Colombo-Peruana, 2011).

Lo anterior es tan obvio en la priorización de proyectos, que se constata durante las reuniones mantenidas por el Comité Técnico Binacional de Desarrollo e Integración Fronteriza (СTBDIF) al interior de la Comisión de Vecindad, que privilegiaron acciones de "desarrollo social", particularmente en el área de salud, como las jornadas binacionales de apoyo, el plan binacional de salud, el plan integral de salud y el proyecto de telemedicina y "desarrollo económico productivo" a través del proyecto de agroforestería, mientras en el componente de "fortalecimiento institucional", se concentró en el Plan de Desarrollo de la zIF y localmente, solo contempló el proyecto "capacitación a autoridades locales en gestión de proyectos (Acta Final. x Reunión de la Comisión de Vecindad e Integración Colombo-Peruana, 2011). Esta línea de acción permanece vigente al interior del CTBDIF, resaltando en su reunión en febrero de 2013, como temas prioritarios, el Plan de Desarrollo de la Zona de Integración Fronteriza y las jornadas binacionales coordinadas por las Fuerzas Armadas de ambos países. 
Lo anterior muestra cómo la agenda bilateral se adelanta de forma descoordinada y poco planificada entre los diferentes niveles de gobierno y expresa un peligro latente para los entes territoriales locales e intermedios en el marco de los procesos de integración, quienes pese a estar presentes en algunos foros de definición de las agendas púbicas, tiene poca injerencia sobre estas. La escasa preparación y participación efectiva tanto de los entes locales e intermedios de gobierno, como de la sociedad civil fronteriza, hace pensar que no se encuentran preparados ni articulados institucionalmente a las condiciones que están tejiéndose en los escenarios bilaterales. Así, los programas que han sido privilegiados tanto en la Comisión de Vecindad, como en el CTBDIF, sin desestimar su importancia como esfuerzo binacional para enfrentar la mantenida precariedad social y productiva de la región, así como para favorecer la conservación de los ecosistemas compartidos, no constituyen mecanismos fortalecedores de la institucionalidad local, dado que no contemplan su participación ni inclusión directa en los citados programas. Por ejemplo, las jornadas binacionales adelantadas por las Fuerzas Navales de ambos países, son un "analgésico" a la precariedad de los sistemas de salud en la frontera. Esto no sugiere acciones mantenidas ni programas permanentes estructurales para la atención en salud; lo mismo ocurre con el programa de telemedicina, el cual sin la infraestructura técnica, personal calificado, mecanismos para realizar traslados de enfermos, vínculos institucionales y esquemas de gestión interadministrativa, tiene un campo de acción limitado. Otros programas en salud, que actualmente se encuentran en fase de estudio como el plan binacional de salud y el plan integral de salud, podrían conducir en el futuro a diseñar acciones institucionales binacionales más coordinadas, sin embargo no se percibe un fortalecimiento efectivo de dichos caminos y mucho menos que las competencias locales se vean reforzadas en el marco de la coordinación binacional.

Pese a la debilidad en la construcción de estructuras institucionales locales y de articulación transfronteriza, se resalta una importante coordinación entre la Comisión Mixta de Cooperación Amazónica del PPCP y la Comisión de Vecindad, toda vez que parecen concurrir en el objetivo de consolidar la zIF entre ambos países, lo cual hace notar una importante continuidad entre los logros alcanzados al interior del PPCP. Sin embargo, las restricciones de la reconfiguración institucional que se vislumbran, parece que no conseguirán romper los amarres nacionales con los cuales funcionó el PPCP y que condujeron a su reformulación durante la IX reunión de la Comisión de Vecindad en 2009 que creó la Comisión de Transición del PPCP hacia la ziF.

Lo anterior se manifiesta al analizar la estructura organizativa que establece el acuerdo al que llegaron las cancillerías en septiembre de 2014 (Acuerdo para la implementación del Plan de Desarrollo de la Zona de Integración Fronteriza entre la República de Colombia y la República del Perú, 2014). Este estableció una Comisión Binacional para la zIF conformada por los ministros de Relaciones Exteriores o sus delegados y los gobernantes de los niveles intermedios de gobiernos: Departamentos de Amazonas y Putumayo y para Perú de la Región de Loreto. Dicha comisión será presidida por los ministros y tendrá una Secretaría Ejecutiva compuesta para Colombia por delegados del Ministerio de Relaciones Exteriores y para Perú por el Consejo Nacional de Desarrollo de Fronteras e Integración Fronteriza — Presidido por el Ministerio de Relaciones Exteriores de Perú- con apoyo del Ministerio de Agricultura y el Pedicp. Por otra parte, existirá un comité local de gestión en el cual participan instituciones regionales y locales, que serán convocadas por la secretaría ejecutiva.

Dicha estructura conduce a pensar que tras las críticas del informe conjunto de las contralorías de ambos países y en contra del clamor de diversos actores locales, la institucionalidad para implementar la zIF sigue siendo un asunto de las cancillerías, 
quienes diseñan e implementan a través de instituciones de carácter nacional, mientras la participación de los niveles intermedios de gobierno es apenas nominal —no tienen funciones asignadas dentro de la estructura funcional de la comisión-. Adicionalmente, las otras entidades presentes en lo local - lo cual incluye las instituciones del orden nacional que operan en el área de la ZIF- apenas pueden hacer "proposiciones" y su participación efectiva en la comisión depende de los deseos e intereses que se jueguen en los ministerios, quienes dominan la dirección y la secretaría ejecutiva del mecanismo. Por su parte, la sociedad local fronteriza, beneficiaria y padecedora de lo que resulte de todo este plan, ni siquiera es mencionada. Entonces, seis años después de que las contralorías comprobaran las restricciones del PPCP y tras doce de que ambos países hayan iniciado el camino de consolidar una zIF, se llegó al mismo punto.

\section{¿Podría la Zona de Integración Fronteriza colombo-peruana superar las restricciones estructurales de los anteriores mecanismos? Una mirada al plan de la Zona de Integración Fronteriza}

Pese a que elementos de la sociedad civil fronteriza (así como representantes del nivel intermedio de gobierno) han estado presentes en diversos foros, al interior de los cuales se definió la agenda bilateral, su visibilidad e injerencia en esta ha sido marginal, siendo privilegiadas las agendas nacionales en la relación, lo cual incluso se hace evidente en la acción binacional en la frontera; cabe preguntarse si el plan de la ZIF enfrenta esta precariedad o reproduce los modelos nacionales en la relación.

El documento de caracterización y diagnóstico de la Zona de Integración Fronteriza elaborado por el Pedicp y entregado en mayo de 2011, así como el documento final del Plan de Desarrollo de la zIF Perú-Colombia entregado finalmente en 2013, identifica estas debilidades, e indica que la Comisión de Transición del PPCP trasladó las inquietudes presentadas en el informe de auditoría de 2008 al Pedicp como entidad consultora, indicándole que debía "prever en el plan de la zIF los mecanismos y procedimientos que permitan la adecuada dinámica institucional y la apropiación de recursos para su implementación y gestión” (Pedicp, 2011, p. 36), así como las directrices de la Ix Reunión de la Comisión de Vecindad e Integración para la formulación del Plan de la zIF que implicaba además de contemplar los estudios previos, "guardar correspondencia entre las iniciativas presentadas en el ámbito de la ZIF con los planes nacionales, departamentales y municipales" (Pedicp, 2011, p. 37). Esto condujo, después de un somero análisis del sistema político-administrativo, a indicar, para la parte colombiana que:

De acuerdo a los distintos análisis sobre el desarrollo institucional en esta parte del país, aún está pendiente la tarea de clarificar, armonizar y consolidar las estructuras y competencias jurídico políticas y administrativas de las entidades territoriales con acción en el territorio de la zIF que permitan en el futuro un verdadero proceso de implementación de la integración y el desarrollo de la zona (Pedicp, 2011, p. 236).

Pese al anterior diagnóstico, la matriz de actores involucrados desarrollada por el Pedicp, proporciona un peso secundario a los niveles intermedios y locales de gobierno y no puntualiza su accionar. Indica en la tabla 6 de su informe, que la gobernación y las alcaldías de Leticia y Puerto Nariño tienen el rol de "definición de programas y 
proyectos a través de planes de desarrollo local y asignación de recursos a proyectos de integración fronteriza", lo cual se ajusta a la normatividad nacional, mientras el Gobierno Regional de Loreto tiene el rol de "orientar el desarrollo regional, plantea y desarrolla estrategias y políticas de desarrollo integrales y sectoriales que le corresponden" (Pedicp, 2011, p. 243) y excluye a los gobiernos locales peruanos como actores directos del plan.

Lo anterior, se enmarca en las funciones explícitas de estos niveles de gobierno. Tanto en Colombia como en Perú, los niveles intermedios y locales tienen funciones de planeación y desarrollo, así como algunas específicas en zonas fronterizas. Por ejemplo, para el caso colombiano, el artículo 289 de la Constitución de 1991 permite a los departamentos y municipios fronterizos adelantar directamente con la entidad territorial limítrofe del país vecino, de igual nivel, programas de cooperación e integración, dirigidos a fomentar el desarrollo comunitario, la prestación de servicios públicos y la preservación del ambiente (Constitución Política de Colombia, 1991, art. 289). Por su parte, la Constitución peruana de 1993 a través del artículo 138, reconoce facultades especiales a las municipalidades de Provincia y Distrito ubicadas en las fronteras, permitiéndoles celebrar protocolos y convenios de integración entre sí, con sus similares nacionales o internacionales, haciendo necesaria para esta última, la participación del Ministerio de Relaciones Exteriores, lo cual, se suma a elementos normados de la descentralización político-administrativa y fiscal, hacia los niveles intermedios y locales de gobierno como a las particularidades de la legislación de fronteras de ambos países (Landa y Velazco, 2007).

En ese sentido, el documento anterior no aporta nada nuevo, solo presenta parcialmente algunas funciones ya normadas, pero no otorga a dichos entes de gobierno canal alguno para articularse de forma eficiente a los instrumentos de acción binacionales como tampoco para establecer relaciones con sus pares al otro lado de la frontera, quedando por tanto en el aire la buena intención de la celebración de convenios transfronterizos a través de la ZIF, constriñéndose a los diseños nacionales al no generar un mecanismo mediante el cual pueda realizarse en lo local una cooperación interadministrativa efectiva.

Dicha tendencia se explicita en el Plan de Desarrollo de la zIF publicado en 2013, donde en pocas ocasiones se hace notorio el fortalecimiento institucional local, pese a que en el objetivo estratégico 3 se referencia transversalmente a la institucionalidad indígena y el objetivo estratégico 6 se concentre en: "fortalecer la institucionalidad pública y privada nacional (regional y local) y binacional de la ZIF" (Pedicp, 2013, pp. 91-92), los objetivos específicos y líneas de acción de estos, se quedan cortos frente a la magnitud del desarrollo de dichos objetivos, no consideran las facultades ya existentes para la institucionalidad local y no se desarrollan claramente a través de los programas y proyectos contemplados en el plan (ver Tabla 2).

Si tomamos en cuenta lo anterior y una revisión mucho más específica de todas las estrategias y líneas de acción del plan de la ZIF, se demuestra que este no apunta al fortalecimiento institucional local entendido como fortalecimiento, consolidación o creación de los entes político-administrativos locales, la cooperación internacional entre ellos o mecanismos de integración transfronteriza. 
Tabla 2: Comparación objetivos estratégicos, líneas de acción y estratégicas del Plan de la ZIF Perú-Colombia

\section{Objetivo estratégico 3}

Fortalecer la identidad cultural de los pueblos indígenas, consolidando sus planes de vida y su interculturalidad a los procesos de desarrollo nacional e integración fronteriza.

\begin{tabular}{|c|c|}
\hline Líneas de acción & Estrategia 1: \\
\hline $\begin{array}{l}\text { Gestión de los planes de vida, conforme a las } \\
\text { políticas nacionales establecidas. }\end{array}$ & \multirow{3}{*}{$\begin{array}{l}\text { Reconocimiento y revalorización de la cultura y las } \\
\text { tradiciones de las comunidades indígenas: } \\
\text { Ideas clave } \\
\text { - Pueblos y comunidades indígenas son sim- } \\
\text { bióticos con el ambiente natural amazónico. } \\
\text { Conocimientos ancestrales, dominio, re- } \\
\text { tomar y ampliar valores y conjugarlos con } \\
\text { conocimientos científicos para avanzar en la } \\
\text { ciencia, la cultura y la relación del hombre } \\
\text { con la naturaleza. }\end{array}$} \\
\hline $\begin{array}{l}\text { Incorporación de medicina tradicional en la } \\
\text { prestación de servicios de salud. }\end{array}$ & \\
\hline $\begin{array}{l}\text { Sobre la naturaleza constituyen patrimonio } \\
\text { invaluable. Su reconocimiento se dirige a: } \\
\text { - } \quad \text { Introducción de modelos educativos que } \\
\text { afiancen la interculturalidad, uso de lenguas } \\
\text { nativas. } \\
\text { Incorporación de conocimiento tradicional } \\
\text { en modelos productivos. }\end{array}$ & \\
\hline
\end{tabular}

\section{Objetivo estratégico 6}

Fortalecer la institucionalidad pública, privada nacional (regional y local) y binacional en la ZIF, que facilite la integración y cooperación entre las entidades públicas, las organizaciones sociales, los pueblos indígenas y los habitantes de frontera, y promueva el empoderamiento local y regional.

\begin{tabular}{|c|c|}
\hline Líneas de acción & Estrategia 1 \\
\hline $\begin{array}{l}\text { Establecimiento de un Sistema de Información } \\
\text { Geográfica en el ámbito de la ZIF, que recoja, siste- } \\
\text { matice y compatibilice la información cartográfica } \\
\text { obtenida de las diversas instituciones que manejan } \\
\text { información relevante para el plan. }\end{array}$ & \multirow{2}{*}{$\begin{array}{l}\text { Fortalecer la presencia de los Estados } \\
\text { en la zIF: } \\
\text { Ideas clave } \\
\text { - Débil presencia estatal en la frontera. } \\
\text { - Intervenir en proveer servicios básicos sufi- } \\
\text { cientes y de calidad. } \\
\text { Mejorar las condiciones de las instituciones } \\
\text { públicas, condiciones laborales y salariales } \\
\text { de funcionarios. } \\
\text { - Fomentar la soberanía alimentaria con apoyo } \\
\text { a productores locales. } \\
\text { Estrategia sujeta a voluntad de los Estados. }\end{array}$} \\
\hline \multirow[t]{2}{*}{$\begin{array}{l}\text { Formulación, adopción y aplicación de la norma- } \\
\text { tividad necesaria para consolidar la integración y } \\
\text { cooperación binacional, con énfasis en el tránsito } \\
\text { de personas, comercio fronterizo y libre navegación } \\
\text { de los ríos comunes. }\end{array}$} & \\
\hline & Estrategia 2 \\
\hline $\begin{array}{l}\text { Promoción y fortalecimiento de las organi- } \\
\text { zaciones comunitarias. }\end{array}$ & \multirow{2}{*}{$\begin{array}{l}\text { Fortalecimiento de actores locales y regionales: } \\
\text { Ideas clave } \\
\text { - Escasa capacidad de crear fuerza de trabajo } \\
\text { sustento de los cultivos para narcotráfico. } \\
\text { Para enfrentar el narcotráfico, desarrollo de } \\
\text { programas lícitos productivos con asistencia } \\
\text { técnica. Se debe aprovechar la biodiversidad } \\
\text { y potencialidades ecológicas con las que } \\
\text { cuenta la región para la formulación de di- } \\
\text { chas alternativas. }\end{array}$} \\
\hline $\begin{array}{l}\text { Gestión de recursos para la ejecución del plan. Ex- } \\
\text { periencia de Sinchi y Pedicp: } \\
\text { - La estrategia está sujeta a voluntad de los } \\
\text { Estados definición y/o conformación de las } \\
\text { instituciones de gestión y ejecución del plan. }\end{array}$ & \\
\hline
\end{tabular}

Fuente: Para objetivo estratégico 3, elaboración propia a partir de Pedicp (2013, pp. 91 y 98). Para objetivo estratégico 6, elaboración propia a partir de Pedicp (2013, pp. 92, 100 y 101). 
Esta tendencia se refuerza en la estructura organizacional del Plan, en la cual las tres opciones enunciadas por la consultora, desplazan a los entes locales e intermedios de gobierno de la coordinación, direccionamiento e implementación de las acciones de la ZIF. Esto se percibe como un mecanismo de desinstitucionalización local, dado que los ejes centrales sobre los cuales se desarrollan la mayoría de los objetivos del Plan, se superponen con las competencias de los entes locales e intermedios de gobierno en ambos países. Es decir, se crea una nueva institucionalidad binacional que actúa por encima de la planificación local y cuyos objetivos se concentran en desarrollar infraestructura social (saneamiento básico, educación y salud) y generar procesos productivos, los cuales corresponden a las funciones que ya tienen los entes locales de gobierno en la legislación de ambos países. Por lo tanto, los Estados tal como lo corrobora la Comisión Binacional para la zIF (Acuerdo para la implementación del Plan de Desarrollo de la Zona de Integración Fronteriza entre la República de Colombia y la República de Perú, 2014) ya analizada, están haciendo un gran esfuerzo binacional para adelantar acciones que deberían cumplirse desde sus propios órganos político-administrativos. ¿No sería más fácil y económico diseñar estrategias para coordinar acciones desde la institucionalidad local vigente? ¿Para qué crear algo que haga lo que los niveles de gobierno actuales ya están obligados a hacer en sus ámbitos de competencia?

\section{Hacia la diplomacia del nivel local y la institucionalidad transfronteriza. Propuestas para dejar de cargar la piedra de Sísifo}

Llegados a este punto, se ha hecho un recorrido por algunos de los mecanismos bilaterales de cooperación e integración que han estado presentes en el intento de implementar una política pública fronteriza para la región de frontera colombo-peruana. Si bien hay una tendencia a la proliferación de objetivos e iniciativas útiles para la región, todas han tenido y siguen teniendo problemas, relacionados con una institucionalidad que termina siendo precaria, ineficiente y limitada, así como excluyente en términos de gestión, diseño e implementación desde los niveles locales e intermedios de gobierno, hasta otros actores de la región fronteriza, cuestionando inclusive las autonomías políticas y administrativas, bastión de la descentralización y sustento de buena parte de la democracia en estos países.

Así las cosas, teniendo presente las dificultades y problemáticas que generan este tipo de mecanismos pensados y administrados desde niveles centrales de gobierno, es importante y necesario, formular alternativas de integración que aprovechen las competencias ya establecidas para los entes territoriales fronterizos de nivel local e intermedio en ambos países, en aras de establecer un nuevo enfoque para la política pública fronteriza en las diferentes regiones de frontera y, en este caso, específicamente para la región fronteriza colombo-peruana.

Un camino diferente en la relación bilateral implicaría pensarse otra Zona de Integración Fronteriza posible. Una zIF donde sean las localidades fronterizas las actoras de sus propios destinos, donde las instituciones de carácter nacional no sean ejecutoras sino asesoras de los caminos del desarrollo. Tal camino es probable si se fortalecen las instancias de coordinación y concertación creadas en ambos países —siendo indispensable la articulación de Brasil a estas iniciativas-, tanto como los recursos y capacidades de gestión de los entes territoriales fronterizos. En esta línea discurren parte de las recomendaciones realizadas por el Grupo de Estudios Transfronterizos en el marco del proyecto "Ciudades y Poblaciones Fronterizas: Una propuesta hacia la armonización 
de las políticas públicas en la frontera internacional del Departamento del Amazonas con Brasil y Perú" (Aponte et al., en prensa), las cuales constituyen una sugerencia para la construcción en un debate amplio sobre una propuesta alternativa para la administración de la frontera en el marco de una Región de Integración Transfronteriza Amazónica (RITA) construida desde lo local.

En términos generales la propuesta contempla que el diseño de las estructuras organizacionales para la administración de las fronteras responda a las demandas locales y no a los deseos nacionales, apoyándose en las instancias que indican tanto en las normativas como las políticas de frontera, cooperación e integración de ambos países. Es decir, que la política de frontera colombo-peruana e inclusive brasileña, debe adelantarse desde los ámbitos locales a través de comisiones trilaterales, donde los niveles centrales de gobierno sean partícipes, sin tener en dicha instancia funciones de dirección, la cual recaería en los niveles intermedios y locales de gobierno. La coordinación de la política sería realizada tanto por un comité de integración trilateral, donde participarían los comités organizados en cada uno de los países y que determinarían las líneas de acción puntuales a ser desarrolladas por los entes territoriales de carácter local de cada uno de los países, en el marco de sus funciones constitucionales. En dicha propuesta, las diversas organizaciones de carácter estatal, supranacional e inclusive no gubernamental, con funciones e intereses en la Amazonia, podrían ser asesoras de la RITA, sin tener funciones de coordinación o dirección, tal como es propuesto en otros esquemas como se observa en la Figura 2.

Figura 2: Organización Administrativa de la Región de Integración Transfronteriza (RITA)

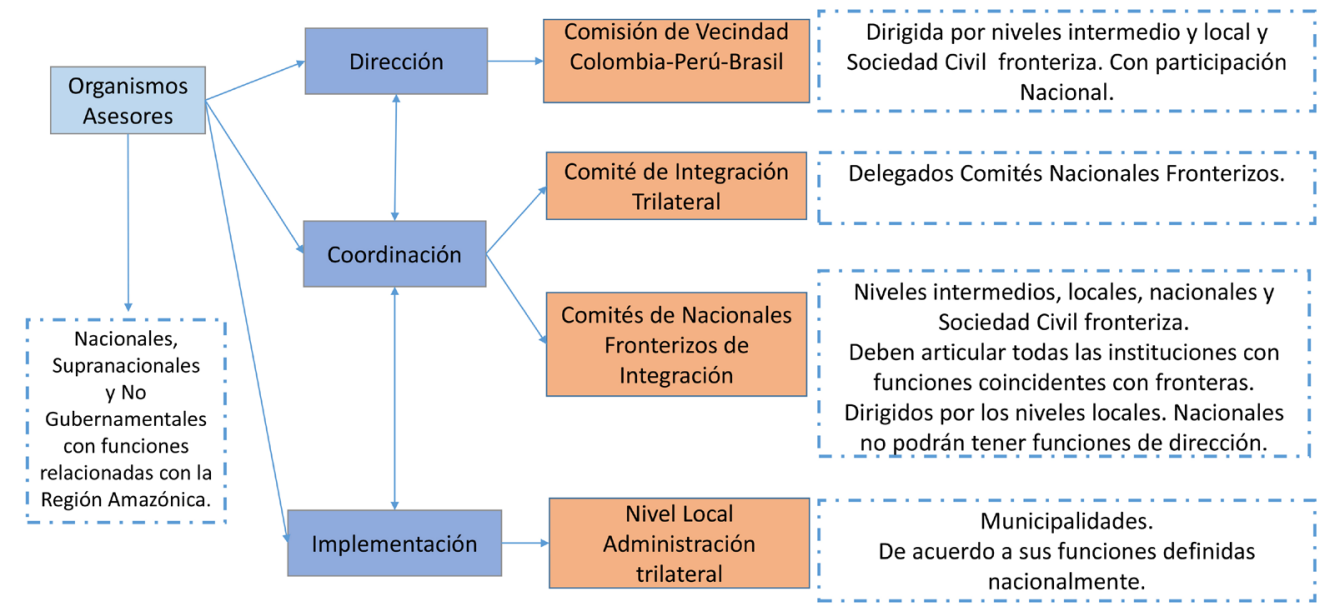

Fuente: Grupo de Estudios Transfronterizos, 2016.

\section{Conclusiones}

Uno de los elementos más profundos de la transformación actual de los Estados nacionales tanto en los planos políticos, como administrativos y fiscales, es que estos ya no son más los contenedores privilegiados de las relaciones sociales, ni los únicos actores del escenario internacional, hoy, pensar la administración local del Estado desde la frontera, implica imaginar otras formas de gobierno, maneras novedosas de administración pública 
de la frontera que ya no pueden moverse en las imaginaciones westfalianas ni en los presupuestos de una geopolítica anquilosada en las nociones realistas de las Relaciones Internacionales. La diplomacia ya no está entre los buenos saberes de un príncipe para relacionarse con otros reinos, tal como lo planteara hace muchos años Nicolás Maquiavelo, sino está, al menos si intentamos constreñirnos a lo que queda de los Estados nacionales, en los mecanismos y estrategias que desarrollan las diferentes formas administrativas político-territoriales, organizaciones sociales y actores que ejercen en y a través de este, para enlazarse con redes y estructuras de intercambio de todo tipo a escalas planetarias, buscando en este camino formas novedosas de gobierno. Es decir, el buen conocimiento de la diplomacia en las condiciones contemporáneas y de las particularidades locales fronterizas necesarias para un gobierno adecuado, democrático, eficiente y responsable, se encuentra mejor ubicado en poblaciones y administraciones públicas locales, que en las grandes capitales que diseñan políticas y acciones nacionales. Quizá esto siempre ha sido así, pero el cambio de las condiciones geopolíticas y geoeconómicas de carácter mundial lo ha hecho aún más evidente.

Colombia y Perú no han sido sordos a tal transformación institucional. De hecho, ambos países han sido ecos de la transformación profunda de los Estados en sus matices más liberalizantes y ambos han liderado el proceso de transformación a nivel supranacional, particularmente al interior de la can. Sin embargo, vemos con cierta preocupación, que tal alborozo descentralizante en la política fronteriza, parece ser más discursivo que práctico, dado que no se traduce en andamiajes institucionales funcionales a tales ideales y tampoco se manifiesta alguna claridad en las presupuestas para financiarlos. Así, de dientes para afuera, tanto las políticas nacionales de frontera como los mecanismos supranacionales de los dos países, son altamente descentralizantes, pero en la práctica interna, siguen atados al centralismo principesco que en las relaciones internacionales marcó la Paz de Westfalia.

Por tanto, la región fronteriza colombo peruana, como Sísifo, sigue cargando la pesada piedra de las estructuras nacionales sin una adaptación clara a las condiciones contemporáneas y las demandas locales. Si no se piensa de otra forma la institucionalidad para la administración de la frontera y la cooperación transfronteriza, el pobre Sísifo seguirá eterna e infructuosamente subiendo la montaña.

\section{Referencias}

Anderson, J., O'Dowd, L., y Wilson, T. M. (2003). New borders for a changing Europe: cross-border cooperation and governance. Londres, Inglaterra: Psychology Press.

Aponte, J., Victorino, N., y Zárate, C. (En prensa). Visibilización, reconocimiento y posicionamiento de una región fronteriza en la Amazonia: Una propuesta hacia la compatibilización e integración de las políticas públicas de frontera de Brasil, Colombia y Perú. Leticia, Colombia: Universidad Nacional de Colombia Sede Amazonia.

Coletti, R. (2010). Cooperación transfronteriza y trayectorias de desarrollo: aprendizajes de la experiencia europea. Si Somos Americanos, 10(1), 154-173.

Ferrero, M. (2006). La globalización en acción: regionalismo y paradiplomacia en Argentina y el Cono Sur latinoamericano. Revista Electrónica de Estudios Internacionales (REEI), (11), 1-22.

García, J. M. R. (2015). Gobiernos locales y la cooperación transfronteriza México-Estados Unidos. Espiral. Estudios sobre Estado y Sociedad, 9(25), 107-140. 
Landa, C. y Velazco, A. (2007). Constitución Política Del Perú 1993: Sumillas. Reformas Constitucionales. Índice analítico. Perú: Pontificia Universidad Católica del Perú, Fondo Editorial.

Ministerio de Relaciones Exteriores. (1994). Comisiones binacionales de vecindad: Comisión Colombo Peruana (Vol. 4). Bogotá, Colombia: Tercer Mundo Editores.

Moreno, S. M. (2012). Fortalecimiento de la gobernanza trans-fronteriza en América Latina a través de la cooperación descentralizada: la experiencia del programa fronteras abiertas. Si Somos Americanos. Revista de Estudios Transfronterizos, 10(1), 147-160.

Oddone, N., y Rodríguez, H. (2015). Cross-border paradiplomacy in Latin America. Latin American Policy, 6(1), 110-123.

Oliveras, X., Durà, A., y Perkmann, M. (2010). Las regiones transfronterizas: balance de la regionalización de la cooperación transfronteriza en Europa (1958-2007). Documents d'anàlisi geogràfica, 56(1), 0021-40.

Peña, S. (2004). Planificación transfronteriza: Instituciones binacionales y bilaterales en la frontera México-Estados Unidos. En S. Tabuenca y T. Payan (Eds.), Gobernabilidad ingobernabilidad en la región paso del norte: Reflexión desde distintas perspectivas (pp. 5984). México: El Colegio de la Frontera Norte, New Mexico State University, Universidad Autónoma de Ciudad Juárez, NEON.

Proyecto Especial para el Desarrollo Integral de la Cuenca del Río Putumayo (Pedicp). (2011). Caracterización y Diagnóstico del Área de Frontera de la Zona de Integración Fronteriza (ZIF) Colombo-Peruana. Iquitos, Perú: Autor.

Proyecto Especial para el Desarrollo Integral de la Cuenca del Río Putumayo (Pedicp). (2013). Plan de Desarrollo de la Zona de Integración Fronteriza Perú-Colombia. Iquitos, Perú: Autor.

Restrepo, D. I. (2001). El mito de Sísifo o 20 años de pujanza descentralizadora. En G. Misas (Ed.), Desarrollo económico y social en Colombia. Siglo xx ( $1^{\mathrm{a}}$ ed., pp. 427-479). Bogotá, Colombia: Universidad Nacional de Colombia, Facultad de Ciencias Económicas.

Sausi, J. L, y Oddone, N. (2010). Cooperación e integración transfronteriza en el Mercosur: el caso de la Triple Frontera Argentina, Brasil y Paraguay. En L. Maira (Ed.), La política internacional subnacional en América Latina (pp. 209-258). Buenos Aires, Argentina: Libros del Zorzal.

Sausi, J. L., y Oddone, N. (2012). Cooperación transfronteriza e integración: Oportunidades para el desarrollo del Perú. Tendencias, 13(1), 239-264.

\section{Material legislativo}

Acta Final. x Reunión de la Comisión de Vecindad e Integración Colombo-Peruana, 2011.

Acuerdo Marco entre la República del Perú y la República Federativa del Brasil para el establecimiento de la "Zona de Integración Fronteriza Perú-Brasil", 2009.

Acuerdo para la implementación del Plan de Desarrollo de la Zona de Integración Fronteriza entre la República de Colombia y la República del Perú, 2014.

Canje de notas que constituye un acuerdo sobre la redefinición del rol y estructura de la Comisión de Vecindad e Integración Colombo Peruana al fin de dotarla de mayor operatividad y agilidad en su funcionamiento entre la república de Colombia y Perú, 2003.

Constitución Política de Colombia de 1991. 
Convenio de Cooperación Aduanera entre la República de Colombia y la República Peruana. (10 de mayo de 1938). Recuperado de http://apw.cancilleria.gov.co/Tratados/adjuntosTratados/PE-10-05-1938.PDF

Decreto 0211 por el cual se crea la Comisión Administradora Nacional del Convenio de Cooperación Aduanera Colombo-Peruana [Decreto de 0211], Diario Oficial No. 40.320 (3 de febrero de 1992). Recuperado de http://ccamazonas.org.co/documentos/decreto211.pdf

Decreto 155 de 2014 Acuerdo entre los Gobiernos de la República de Colombia y de la Federativa de Brasil para el Establecimiento de la Zona de Régimen Especial Fronterizo para las localidades de Tabatinga (Brasil) y Leticia (Colombia). Recuperado de http://wsp.presidencia.gov.co/Normativa/Decretos/2014/Documents/FEBRERO/05/DECRETO \% 20155\%20DEL \% 2005\%20DE\% 20FEBRERO \% 20DE\% 20 2014.pdf

Decreto 2791 Por medio del cual se modifica el Decreto 2830 del 21 de diciembre de 2001, Diario Oficial No. 45.329 (3 de octubre de 2003). Recuperado de https://www.cancilleria.gov.co/sites/default/files/Normograma/docs/decreto_2791_2003.htm

Decreto 3157 Por medio del cual se modifica el Decreto 2791 de 2 de octubre de 2003, Diario Oficial No. 46.730 (23 de agosto de 2007). Recuperado de http://www.cancilleria.gov.co/sites/default/files/Normograma/docs/decreto_3157_2007.htm

Informe Internacional de la Auditoría en Cooperación. Informe de auditoría. Plan Colombo Peruano para el Desarrollo Integral de la Cuenca del Río Putumayo-PPCP-1998-2008. (2008). Recuperado de http://www.environmental-auditing.org/Portals/0/AuditFiles/Colombia_Peru_08_f_eng_PPCP.pdf

Ley 1463 de 2011 Acuerdo entre los Gobiernos de la República de Colombia y de la Federativa de Brasil para el establecimiento de la Zona de Régimen Especial Fronterizo para las localidades de Tabatinga (Brasil) y Leticia (Colombia), Diario Oficial No. 48.116. Recuperado de http://www.secretariasenado.gov.co/senado/basedoc/ ley_1463_2011.html

Plan Colombo-Peruano para el Desarrollo Integral de la Cuenca del Río Putumayo-Diagnóstico Regional Integrado (PPCP). (1993). Recuperado de http://www.oas.org/ dsd/publications/unit/oea62s/oea62s.pdf

Plan Colombo-Peruano para el Desarrollo Integral de la Cuenca del Río Putumayo (PPCP). (1998). Recuperado de http://www.oas.org/usde/publications/Unit/oea82s/begin.htm\#Contents

Protocolo de amistad y cooperación entre la república del Colombia y la República del Perú y Acta Adicional, art. 4, 1934.

Protocolo de amistad y cooperación entre la república del Colombia y la República del Perú y Acta Adicional, art. 14 del acta adicional, 1934.

Protocolo Modificatorio del Convenio de Cooperación Aduanera Colombo-Peruano de 1938, art. IX, 1981.

Adriana Mayela Hurtado

Colombiana. Finalizó sus estudios de Pregrado en Ciencia Política de la Universidad Nacional de Colombia. Es investigadora del Grupo de Estudios Transfronterizos de la Universidad Nacional de Colombia Sede Amazonia y se encuentra vinculada a la Escuela de Gobierno y Políticas Públicas de la Universidad de Strathclyde en Glasgow, Reino 
Unido. Líneas de investigación: la implementación de políticas públicas, análisis de fronteras y relaciones internacionales. Publicaciones recientes en 2013: El desarrollo fronterizo: El concepto clave para las fronteras. En C. Chacón (Ed.), Política y relaciones internacionales colombianas: Una mirada a la gestión 2012 (Vol. 1, pp. 11-23).

Jorge Aponte Motta

Colombiano. Politólogo por la Universidad Nacional de Colombia, Maestro en Estudios Amazónicos Sede Amazonia y candidato a Doctor en Geografía por Universidad Autónoma de Madrid (UAM). Investigador en Formación del Departamento de Geografía de la UAM e investigador del Grupo de Estudios Transfronterizos de la Universidad Nacional de Colombia Sede Amazonia. Líneas de trabajo: estudio de las dinámicas urbanas y fronterizas en la región amazónica. Entre sus publicaciones recientes se encuentra: Tabatinga como espacio de excepción: La ciudadanía fronteriza y la condición de extranjero a debate. En T. Schor (Ed.), Geografias e cidades na triplice fronteira Brasil-Peru-Colombia (Vol. 3). 